\title{
Using recursive partitioning to account for parameter heterogeneity in multinomial processing tree models
}

\author{
Florian Wickelmaier $^{1}$ • Achim Zeileis ${ }^{2}$
}

Published online: 4 August 2017

๑) Psychonomic Society, Inc. 2017

\begin{abstract}
In multinomial processing tree (MPT) models, individual differences between the participants in a study can lead to heterogeneity of the model parameters. While subject covariates may explain these differences, it is often unknown in advance how the parameters depend on the available covariates, that is, which variables play a role at all, interact, or have a nonlinear influence, etc. Therefore, a new approach for capturing parameter heterogeneity in MPT models is proposed based on the machine learning method MOB for model-based recursive partitioning. This procedure recursively partitions the covariate space, leading to an MPT tree with subgroups that are directly interpretable in terms of effects and interactions of the covariates. The pros and cons of MPT trees as a means of analyzing the effects of covariates in MPT model parameters are discussed based on simulation experiments as well as on two empirical applications from memory research. Software that implements MPT trees is provided via the mpttree function in the psychotree package in $\mathrm{R}$.
\end{abstract}

Keywords Multinomial processing tree models . Model-based recursive partitioning $\cdot$ Parameter heterogeneity

Florian Wickelmaier

florian.wickelmaier@uni-tuebingen.de

1 Department of Psychology, University of Tübingen, Schleichstr. 4, 72076 Tübingen, Germany

2 Department of Statistics, Faculty of Economics and Statistics, Universität Innsbruck, Universitätsstr. 15, 6020 Innsbruck, Austria

\section{Introduction}

Multinomial processing tree (MPT) models are a class of statistical models for categorical data. These models are associated with a graph resembling a probability tree, the links being the parameters, the leaves being the response categories. The path from the root to one of the leaves represents the latent cognitive processing steps a participant executes to arrive at a given response. Since they were introduced in a seminal article (Riefer \& Batchelder, 1988), MPT models have been applied in numerous ways in cognitive psychology and in related fields (Batchelder \& Riefer, 1999; Erdfelder et al., 2009).

As an example, consider an experimental paradigm prevalent in memory research for investigating recognition memory. A recognition-memory experiment consists of two phases: In the learning phase, participants are presented with a list of items to be memorized. In the test phase, old items are presented intermixed with new distractor items, and participants have to classify them as either old or new. Figure 1 displays the structure of the one-highthreshold (1HT) model of recognition (Blackwell, 1963; Swets, 1961), possibly one of the simplest MPT models. According to this model, an old item is recognized as old with probability $r$, or, if not recognized, it is guessed that it is old with probability $b$. Therefore, on the left-hand side of the figure, there are two paths leading from the root of the tree to an old response. Alternatively, displayed on the righthand side of the figure, a new item can only be guessed as being old with probability $b$ since, according to the model assumptions, such an item never exceeds the recognition threshold.

Frequently, it is the goal of a study to investigate the effects of explanatory variables on the parameters of an 
MPT model. Such variables may include experimentally manipulated variables, observed predictor variables, or nuisance variables. In the remainder, we do not distinguish among these and refer to them jointly as covariates. In order to include such covariates, the classical approach is to apply the model to multiple groups defined by these variables and to test for effects (see, e.g., Riefer \& Batchelder, 1991, who study age effects on memory processes). More recently, various approaches to account for parameter heterogeneity have been developed; these include latent-class (Klauer, 2006) and hierarchical MPT models (Klauer, 2010; Smith \& Batchelder, 2010; Matzke, Dolan, Batchelder, \& Wagenmakers, 2015). The latter may be employed to study covariate effects: When the influence of the covariates is linear, they can be directly included in a hierarchical model via specific link functions (Coolin, Erdfelder, Bernstein, Thornton, \& Thornton, 2015; Michalkiewicz, Coolin, \& Erdfelder, 2013; Oravecz, Anders, \& Batchelder, 2015). More broadly, covariate effects represent a form of parameter heterogeneity: Different settings of covariates may lead to a change in model parameters. The automatic detection of such changes is at the heart of our method.

In this paper, we introduce MPT trees, a novel approach to incorporating covariates into MPT models. The main difference of our approach and other existing methods for including covariates is that with MPT trees, the relationship between groups and covariates does not have to be fully specified but is "learned" from the data. The core of this approach is model-based recursive partitioning (Zeileis, Hothorn, \& Hornik, 2008), a tree-based computational method from machine learning for detecting parameter heterogeneity across covariates in a data-driven way. The result is a tree-based classification of all individuals into groups where the MPT model parameters are homogeneous within each group but heterogeneous across groups. Thus, not only do MPT trees test for the presence of parameter heterogeneity, but they also capture it (if any) in interpretable groups without the need for pre-specification of the relevant covariates or their interactions. Some patterns of heterogeneity are easier to detect than others for MPT trees, and we will address their strengths and limitations by simulation studies and in the Discussion section.

For illustration, Fig. 2 depicts an artificial data set following such a tree. In this data set, the responses of all participants are represented by the 1HT model from Fig. 1, but the model parameters vary between three groups that are defined in terms of two covariates, $x_{1}$ and $x_{2}$. A conceivable situation would be a recognition experiment where $x_{1}$ could be an IQ test score (e.g., Fagan, 1984) and $x_{2}$ could be the amount of training with the task. The interpretation would then be: The recognition probability $r$ is lowest for participants with lowest IQ scores as measured in $x_{1}$ (below some threshold or cutoff $v_{1}$ ), whereas those

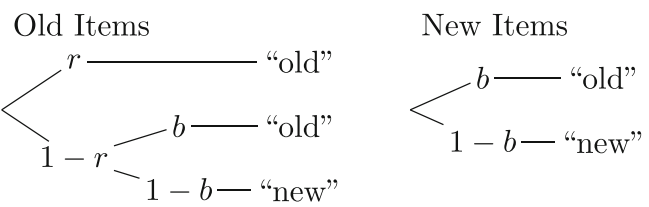

Fig. 1 Graph of the one-high-threshold model for recognition memory (Blackwell, 1963; Swets, 1961). Latent cognitive processes are: recognition of an old item $(r)$ and guessing that a not recognized item is old $(b)$

with higher IQ scores have a higher recognition probability $r$, which even increases further with sufficient training $x_{2}$ above some threshold $\nu_{2}$. In this artificial data set, the guessing probability $b$ is the same across all groups.

Note that this MPT tree combines two levels of trees. The first level is the tree of the MPT model (Fig. 1). Its tree structure has to be specified in advance and is assumed to be constant in the entire population; the parameters ( $r$ and $b$ ) associated with its links, however, are allowed to vary and need to be estimated. The second level is the recursive partitioning based on the subject covariates (Fig. 2). It does not have to be specified in advance but is learned based on the available data. Specifically, neither the correct order of the variables $x_{1}$ and $x_{2}$ nor their cutoffs $v_{1}$ and $v_{2}$ have to be pre-specified but are estimated from the data by modelbased recursive partitioning.

The remainder of this paper is organized as follows: First, the steps of the model-based recursive partitioning algorithm for MPT models are outlined. Next, the performance of the method is investigated in a simulation study based on the artificial scenario from Fig. 2. In a second simulation study, we assess how MPT trees compare to latent-class (mixture) models that are based on the same MPT model. Then, the use of recursive partitioning for investigating effects of covariates on cognitive processes is illustrated with two examples from memory research. Finally, our approach is discussed in the context of other methods for

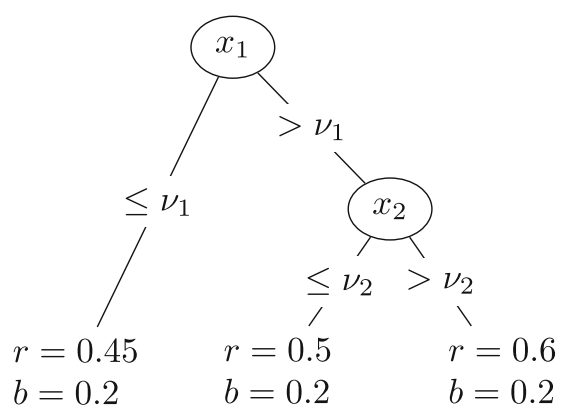

Fig. 2 Tree structure for the artificial data. Two covariates $\left(x_{1}, x_{2}\right)$ along with their binary cutoffs $\left(v_{1}, v_{2}\right)$ define three groups with specific $r$ parameters of the one-high-threshold model 
incorporating covariates or for detecting parameter heterogeneity in MPT models. We conclude by briefly describing the software that estimates MPT trees.

\section{Recursive partitioning based on MPT models}

Model-based recursive partitioning (MOB; Zeileis et al., 2008) is a general approach to account for heterogeneity in parametric models. The basic idea of MOB is that the fit of a model may be improved by splitting the sample and fitting the model to subgroups. These subgroups are formed automatically: The algorithm learns the optimal partitions using the covariates available. Thus, by recursively partitioning the sample, MOB seeks to explain parameter heterogeneity, which is also called parameter instability in the machinelearning context, by means of main effects and interactions of subject covariates.

There already exist adaptations of the MOB algorithm to (multivariate) linear and generalized linear models (Zeileis et al., 2008), to the Bradley-Terry-Luce choice model (Strobl, Wickelmaier, \& Zeileis, 2011), and to the Rasch model and other psychometric models from item response theory (Komboz, Strobl, \& Zeileis, 2016; Strobl, Kopf, \& Zeileis, 2015). Common to these adaptations are the general steps of the MOB algorithm, which are, in summary, as follows:

1. Fit a parametric model to the current (sub-)sample, starting with the full sample, by estimating its parameters via maximum likelihood.

2. Assess the stability of the model parameters with respect to each available covariate. This is done using a parameter instability test based on the maximum likelihood scores.

3. If there is significant instability, select the covariate associated with the strongest instability. Compute the cutpoint that leads to the greatest improvement in the model's likelihood. Split the sample.

4. Repeat steps 1 to 3 until there is no more significant parameter instability or until the minimum sample size is reached.

Thus, all steps are based on the model's likelihood, and the size of the resulting tree is controlled by significance tests. An optional final step, especially for large data sets, is pruning: Splits that do not improve the model fit according to information criteria such as AIC (Akaike, 1974) or BIC (Schwarz, 1978) are omitted from the tree.

In this paper, we will introduce MPT trees, an adaptation of model-based recursive partitioning to MPT models. In the following, the steps of the algorithm specific to MPT models are explained. For the general procedure of modelbased recursive partitioning we refer to Zeileis et al. (2008).

\section{Likelihood of MPT models}

The data consist of the response frequencies for each of $i=1, \ldots, n$ participants in each of $j=1, \ldots, J$ response categories. Let $y_{i}=\left(y_{i j}\right)$ be the vector of observed frequencies for participant $i$ in the response categories. Let $\Theta=\left(\vartheta_{k}\right), k=1, \ldots, K, \Theta \in[0,1]^{K}$, be the vector of MPT model parameters. The MPT model defines the probability of a response in each category, $p_{j}=p_{j}(\Theta)$, as a function of the parameters. Assuming independence of the responses, the data follow a multinomial distribution. The joint likelihood becomes

$$
L\left(\Theta ; y_{1}, \ldots, y_{n}\right)=\prod_{i=1}^{n}\left(y_{i+} ! \prod_{j=1}^{J} \frac{p_{j}(\Theta)^{y_{i j}}}{y_{i j} !}\right)
$$

where $y_{i+}=\sum_{j=1}^{J} y_{i j}$, and it only depends on the MPT model parameters $\Theta$. The kernel of the log-likelihood is proportional to

$\log L\left(\Theta ; y_{1}, \ldots, y_{n}\right) \propto \sum_{i=1}^{n} \sum_{j=1}^{J} y_{i j} \log p_{j}(\Theta)=\sum_{i=1}^{n} \ell\left(\Theta ; y_{i}\right)$,

where $\ell\left(\Theta ; y_{i}\right)$ denotes the log-likelihood contribution of the $i$-th person.

For example, in the recognition-memory experiment introduced above, items are either old or new, and participants have to classify them as old or new in a recognition test. Therefore, the responses of an individual fall into one of $J=4$ categories, resulting in a two-by-two table of response frequencies:

$$
\begin{array}{rrr} 
& \multicolumn{3}{c}{\text { Response }} \\
\text { old } & \text { new } \\
\text { Item old } & y_{i 1} & y_{i 2} \\
\text { new } & y_{i 3} & y_{i 4}
\end{array}
$$

The 1HT model (Fig. 1) has two parameters, $\Theta=(r, b)$, and the predicted probabilities for each response category are

$$
\begin{array}{ll}
p_{1}(\Theta)=r+(1-r) b & p_{2}(\Theta)=(1-r)(1-b) \\
p_{3}(\Theta)=b & p_{4}(\Theta)=1-b .
\end{array}
$$

Thus, the log-likelihood contribution of the $i$-th person becomes

$$
\begin{aligned}
\ell\left(\Theta ; y_{i}\right)= & y_{i 1} \log (r+(1-r) b)+y_{i 2} \log ((1-r)(1-b)) \\
& +y_{i 3} \log b+y_{i 4} \log (1-b) .
\end{aligned}
$$

The sum over all persons' log-likelihood contributions amounts to the kernel of the joint log-likelihood in Eq. 2.

Many prevalent MPT models consist of multiple category systems, or subtrees. For example, the 1HT model has two response categories for old items and two for new items. 
Thus, technically, the corresponding likelihood is product (or joint) multinomial. For parameter estimation and for the instability tests presented below, however, this distinction is irrelevant, so we keep the simplified notation of $J$ categories in total.

\section{Maximum likelihood estimation}

Maximum likelihood estimates of MPT model parameters are obtained by maximizing Eq. 2 with respect to $\Theta$,

$\hat{\Theta}=\underset{\Theta}{\arg \max } \sum_{i=1}^{n} \ell\left(\Theta ; y_{i}\right)$.

One way of solving Eq. 5 is by means of the expectationmaximization (EM) algorithm described in $\mathrm{Hu}$ and Batchelder (1994). The idea is that parameter estimation would be simplified if not only the category frequencies were known but also the frequencies of every single branch from the root to the leaves. The latter are missing, of course, but their expected value can be computed given initial parameter values (E step). With the expected branch frequencies at hand, the parameter values are updated ( $\mathrm{M}$ step). These two steps are iterated until the likelihood converges to a local maximum.

A prerequisite for the application of the EM algorithm is that the link probabilities in a branch take the form

$\gamma \prod_{k=1}^{K} \vartheta_{k}^{\alpha}\left(1-\vartheta_{k}\right)^{\beta}$,

where $\alpha, \beta \in\{0,1\}$ indicate the occurrence of either $\vartheta_{k}$ or $1-\vartheta_{k}$, and $\gamma$ is a nonnegative real number. Equation 6 is the structural restriction of the class of MPT models that can be represented by binary trees. Other model types have to be suitably reparameterized for the algorithm to apply. The 1HT model with the equations in Eq. 3 fulfils the requirement in Eq. 6. Accordingly, the graph of this model and of the models presented in the application section are examples of binary trees.

An alternative way of solving Eq. 5 is by directly maximizing the log-likelihood using analytical gradients (Riefer \& Batchelder, 1988). When doing so, it is advantageous to transform the parameters to the logit scale in order to remove the $[0,1]$ boundaries.

Confidence intervals are straightforward since both parameter estimation methods lead to analytical expressions for the observed Fisher information or negative Hessian matrix (Hu \& Batchelder, 1994, Equation 16; Riefer \& Batchelder, 1988, Equation 21). When working on the logit scale, the information matrix may be obtained by the multivariate delta method (Agresti, 2002; Bishop, Fienberg, \& Holland, 1975; Grizzle, Starmer, \& Koch, 1969). The approximate covariance matrix is available via the inverse information matrix.
Once the model is fit to the full sample, we want to test for parameter heterogeneity that can be attributed to the covariates; this is described next.

\section{Detection of parameter instability}

In the framework of model-based recursive partitioning, a test of parameter instability checks if the model fit can be improved by splitting the sample according to some covariate $X$ and fitting the model to the subsamples. Under the null hypothesis of parameter homogeneity (or stability), it is assumed that Eq. 1 holds $^{1}$ and thus the parameter vector is equal for all participants,

$H_{0}: \Theta_{i}=\Theta_{0} \quad(i=1, \ldots, n)$,

where $\Theta_{i}$ is the parameter vector of individual $i$. The alternative hypothesis is that the parameter vector varies as a function of $X$ with observations $x_{1}, \ldots, x_{n}$,

$H_{1}: \Theta_{i}=\Theta\left(x_{i}\right) \quad(i=1, \ldots, n)$.

The exact pattern of variation is usually unknown. For unordered categorical $X$, such as medical diagnosis or experimental condition, it is common to test for differences in the parameter vector for all categories of $X$. For continuous and ordinal $X$, one frequent pattern of interest is an abrupt change in the parameter vector at an unknown cutpoint $v$,

$H_{1}^{*}: \Theta_{i}= \begin{cases}\Theta^{(A)} & \text { if } x_{i} \leq v, \\ \Theta^{(B)} & \text { if } x_{i}>v,\end{cases}$

where $\Theta^{(A)} \neq \Theta^{(B)}$ (Merkle \& Zeileis, 2013; Merkle, Fan, \& Zeileis, 2014). Possible examples of such a pattern include hypothetical effects of age, expertise, intelligence, etc., where the parameter vector changes at some value $v$.

To test the above hypotheses, the parameter instability statistics employed here make use of the individual contributions to the score function or subject-wise estimating function, $s\left(\Theta ; y_{i}\right)$, and assess the deviations from its mean zero. For MPT models, due to the multinomial form of the likelihood, the contribution of individual $i$ to the score function is given by

$s\left(\Theta ; y_{i}\right)=\frac{\partial \ell\left(\Theta ; y_{i}\right)}{\partial \Theta}=\sum_{j=1}^{J} y_{i j} \frac{\partial \log p_{j}}{\partial \Theta}=\sum_{j=1}^{J} \frac{y_{i j}}{p_{j}(\Theta)} \frac{\partial p_{j}}{\partial \Theta}$.

\footnotetext{
${ }^{1}$ If the model is misspecified and misspecification is associated with some of the partitioning variables, the instability tests may become progressive.
} 
For example, in the 1HT model, the individual score contributions are determined by first partially differentiating the probabilities in Eq. 3 with respect to $\Theta=(r, b)$; this yields

$$
\begin{array}{ll}
\frac{\partial p_{1}}{\partial \Theta}=\left(\begin{array}{l}
1-b \\
1-r
\end{array}\right) & \frac{\partial p_{2}}{\partial \Theta}=\left(\begin{array}{c}
b-1 \\
r-1
\end{array}\right) \\
\frac{\partial p_{3}}{\partial \Theta}=\left(\begin{array}{l}
0 \\
1
\end{array}\right) & \frac{\partial p_{4}}{\partial \Theta}=\left(\begin{array}{c}
0 \\
-1
\end{array}\right) .
\end{array}
$$

Second, substituting these terms into Eq. 10 gives

$s\left(\Theta ; y_{i}\right)=\left(\begin{array}{l}\frac{y_{i 1}(1-b)}{r+(1-r) b}+\frac{y_{i 2}(b-1)}{(1-r)(1-b)}+y_{i 3} \cdot 0+y_{i 4} \cdot 0 \\ \frac{y_{i 1}(1-r)}{r+(1-r) b}+\frac{y_{i 2}(r-1)}{(1-r)(1-b)}+\frac{y_{i 3}}{b}-\frac{y_{i 4}}{1-b}\end{array}\right)$.

The score contributions behave like residuals and are diagnostic of the model fit. Evaluation of the score function for each individual at the joint maximum likelihood estimate $\hat{\Theta}$ measures the extent to which the model maximizes each individual's likelihood: Scores further from zero indicate that the model provides a poorer description of such individuals. The general idea of the tests applied here is that under the null hypothesis of parameter homogeneity Eq. 7, the individual score contributions, when ordered by any covariate $X$, fluctuate randomly around zero. When parameters are not homogeneous across the entire sample, however, the scores systematically depart from zero.

The left panel of Fig. 3 shows the maximum likelihood scores (Eq. 12) for the $r$ parameter of the 1HT model based on a hypothetical scenario as depicted in Fig. 2. The scores when ordered by covariate $x_{1}$ (which might represent, say, an IQ test score) do not fluctuate randomly around zero, but are mostly negative until $x_{1}$ reaches a certain cutpoint, and mostly positive afterwards; this cutpoint is approximately at zero. This suggests that a model with a single $r$ parameter would overestimate $r$ for participants with low IQ scores and underestimate $r$ for participants with high IQ scores.

To capture these deviations, the cumulative score process

$$
B(t ; \hat{\Theta})=\hat{I}^{-1 / 2} n^{-1 / 2} \sum_{i=1}^{\lfloor n \cdot t\rfloor} s\left(\hat{\Theta} ; y_{(i)}\right) \quad(0 \leq t \leq 1),
$$

is employed, where $\lfloor n \cdot t\rfloor$ is the integer part of $n \cdot t, \hat{I}$ is an estimate of the covariance matrix of the scores, and $y_{(i)}$ denotes that $y_{i}$ has been ordered by $X$. Since the sampling distribution of this process under the null hypothesis is known, critical values and $p$ values can be derived either analytically or by simulation. The exact form of the test statistic depends on whether the covariate is continuous, categorical, or ordinal. The right panel of Fig. 3 shows the cumulative score process (Eq. 13) for the $r$ parameter of the 1HT model based on artificial data. Because the scores are mostly negative before and positive after the cutpoint, the cumulative score process has a characteristic triangular shape deviating strongly from the random pattern of a Brownian bridge expected under the null hypothesis of parameter homogeneity.

The tests employed to detect parameter heterogeneity are generalized Lagrange multiplier (LM) tests, also known as score tests. More background information on these tests than provided here is included in several recent articles: Details of the parameter instability tests are discussed by Zeileis and Hornik (2007), who show that they are not restricted to maximum likelihood scores but also apply to other maximum-likelihood-type estimators (M-estimators), like ordinary least squares. Details of the recursive application of these tests and of the model-based recursive partitioning algorithm in general are given by Zeileis et al. (2008). Merkle and Zeileis (2013) discuss the tests in the context of measurement invariance with respect to structural equation models. Merkle et al. (2014) extend the results to ordered categorical covariates.
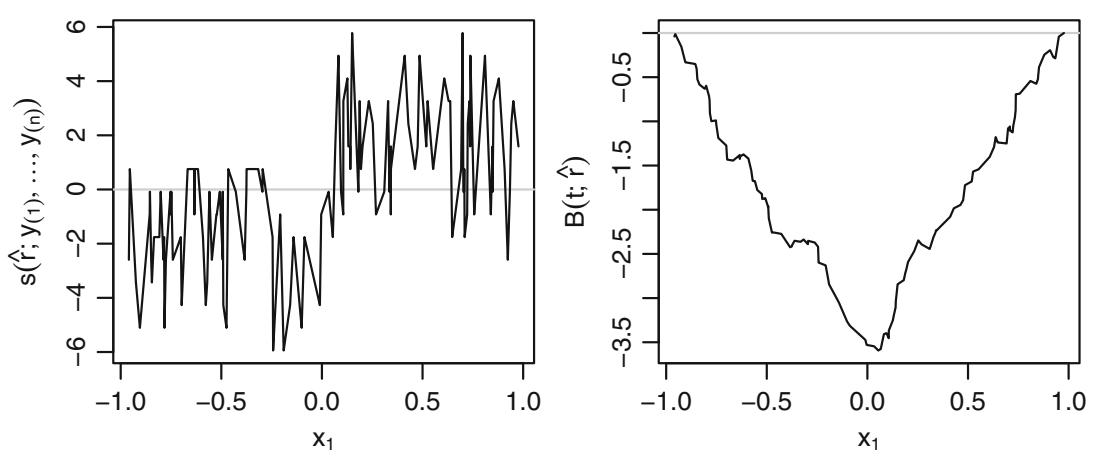

Fig. 3 Maximum likelihood scores (left panel) and cumulative scores (right panel) for the $r$ parameter of the one-high-threshold model based on a hypothetical scenario as depicted in Fig. 2 (artificial data). Both panels indicate a change of the parameter value at a cutpoint in $x_{1}$ around zero 


\section{Cutpoint location and recursive partitioning}

When all available covariates have been tested for parameter instability using the procedure outlined above and at least one test is significant, the MOB algorithm selects the variable that induces the strongest instability (with the smallest $p$ value) in order to locate the cutpoint for splitting the sample. As each test and $p$ value depend on only one of the covariates, taking the minimum $p$ value is invariant against the order in which the covariates are presented to the algorithm. For continuous and ordinal covariates, the idea behind the estimation of the optimal cutpoint $v$ is to find the value of the selected covariate with $x_{m} \leq v$ and $x_{m+1}>v$ that splits the current sample such that the likelihood in the two subsamples

$\ell\left(\hat{\Theta}^{(A)} ; y_{i}, \ldots, y_{m}\right)+\ell\left(\hat{\Theta}^{(B)} ; y_{m+1}, \ldots, y_{n}\right)$

is maximized. For unordered categorical covariates, all possible binary partitions are computed and the one with the maximum segmented likelihood is chosen. Note that for covariates with more than 15 unordered categories, such an exhaustive search will become increasingly computationally expensive and may require parallel computing facilities to terminate within a practically useful time limit.

Once the optimal cutpoint is located and the sample is split, the instability tests are recursively conducted in the two subsamples until there is no further significant instability. Depending on the size of the parameter differences, situations may occur where two tests lead to almost the same $p$ value. Then, small perturbations in the data may (or may not) lead to different trees. Resampling is one common approach for assessing the stability of tree structures (Philipp, Zeileis, \& Strobl, 2016). This is, however, not pursued in the present paper.

Within model-based recursive partitioning, there are two built-in mechanisms that prevent inflation of the type I error rate and, consequently, that a tree grows unwarrantedly large: (1) When testing for instability in a subsample, Bonferroni correction is applied. Thus, instability tests become increasingly strict with an increasing number of covariates. (2) Testing proceeds in a nested fashion, that is, only if a test is significant in a subsample will testing continue in nested subsamples. As a consequence of (1) and (2), a tree does not exceed the nominal significance level $\alpha$ (Zeileis et al., 2008). We will address the statistical performance of the proposed procedure in a simulation study presented next.

\section{Simulation study 1: Power and classification accuracy}

This section describes a simulation study to investigate power, type I error rate, and classification accuracy of MPT trees. The focus of this simulation is restricted to one specific MPT model that is observed under realistic magnitudes of parameter instability and moderate sample sizes. Further complementary simulation results have been reported elsewhere and include power and type I error of score tests for measurement invariance in the context of structural equation modeling (Merkle \& Zeileis, 2013; Merkle et al., 2014), performance of recursive partitioning and comparison to mixture models for linear regression (Frick, Strobl, \& Zeileis, 2014a), performance of Rasch, partial credit, and rating scale trees for detecting differential item functioning (Komboz et al., 2016; Strobl et al., 2015).

\section{Simulation design and experimental settings}

In order to simulate responses, the 1HT model (see Fig. 1) is employed as the data-generating process with groupspecific $r$ parameters and a constant $b$ parameter, $\Theta=$ $\left(r_{\text {group }}, b=0.2\right)$ for group $\in\{1,2,3\}$, see Fig. 2 . Each virtual subject contributes 40 simulated responses (to 20 old and 20 new items). Three subject-specific covariates $\left(x_{1}\right.$, $\left.x_{2}, x_{3}\right)$ are included that are independently uniformly distributed in the interval $[-1,1]$. The interaction between $x_{1}$ and $x_{2}$ along with the corresponding binary cutoff values $v_{1}$ and $v_{2}$ defines three groups: $x_{1} \leq v_{1}$ versus $x_{1}>v_{1} \wedge x_{2} \leq$ $v_{2}$ versus $x_{1}>v_{1} \wedge x_{2}>v_{2}$. The noise variable $x_{3}$ is unrelated to the groups.

The magnitude of parameter instability is controlled by the deviation $\delta \in\{0, .01, .02, \ldots, .20\}$ from the average recognition probability $r=.5$. The group-specific recognition probabilities are $r_{1}=.5-\delta / 2, r_{2}=.5$, and $r_{3}=.5+\delta$. Thus, $\delta=0$ corresponds to parameter homogeneity across the three groups with $r_{1}=r_{2}=r_{3}=.5$. The setup with $\delta=0.1$ is shown in Fig. 2. Moreover, three small-tomoderate sample sizes $n \in\{80,120,200\}$ are considered. We expect that increasing both the magnitude $\delta$ and the number $n$ of participants will lead to improved detection performance of the MPT trees.

Two scenarios are considered for the cutoffs $v_{1}$ and $\nu_{2}$ : First, the median value of the distributions of $x_{1}$ and $x_{2}$ is used, that is, $v_{1}=v_{2}=0$, so that on average the group sizes are $1 / 2,1 / 4$, and $1 / 4$, respectively, of the total sample. Second, $v_{1}=-.5$ and $v_{2}=.5$ are used as the cutoffs resulting in group sizes of about $1 / 4,9 / 16$, and $3 / 16$, respectively. Thus, in the latter scenario, the parameter differences are harder to detect because the middle group (with $r_{2}=.5$ ) is the largest and the deviating groups are smaller.

For benchmarking the power and the accuracy of MPT trees (see below for details on the outcome measures), the frequently used likelihood ratio test (LRT) is employed as a reference method. Because the LRT requires a pre-specified split into groups, we consider the common strategy of splitting at the median of a relevant covariate. Here, we consider 
splitting either $x_{1}$ or $x_{2}$ at their corresponding medians. Note that this gives the LRT a somewhat unfair advantage, especially in the first scenario where the true cutoffs are at the median of zero. Also, the irrelevant covariate $x_{3}$ is not considered at all and no Bonferroni correction is applied for aggregating multiple LRTs.

In summary, for each of the two cutoff scenarios and each combination of magnitude of parameter instability and sample size, 2000 data sets are generated to compute the outcome measures below for the MPT tree method, the LRT with splitting at the median of $x_{1}$, and the LRT with splitting at the median of $x_{2}$, respectively. All simulations were run in $\mathrm{R}$ using software described in the "Computational details and software" section.

\section{Outcome measures}

Two kinds of outcome measures are considered: (1) the power with which the MPT tree and the two LRTs reject the null hypothesis of parameter stability; (2) the accuracy with which the true groups were recovered. For the MPT tree, the power is the proportion of experiments in which the score test in the root node is significant for $x_{1}$ or $x_{2}$, that is, in which the sample is split at least once. For comparison, the power of the two LRTs is the proportion of experiments in which the null hypothesis of $r_{x_{1} \leq 0}=r_{x_{1}>0}$ or $r_{x_{2} \leq 0}=r_{x_{2}>0}$, respectively, is rejected. Note that the hypothesized cutoff value of zero, the median of $x_{1}$ and $x_{2}$, either coincides with the true cutoff (first cutoff scenario) or differs (second cutoff scenario).
The classification accuracy for MPT trees is assessed using the Cramér coefficient of agreement defined as the normalized $\chi^{2}$ statistic of the crosstabulated true and predicted group membership (Mirkin, 2001). It takes a value of zero if the true and predicted groups are uncorrelated, and a value of one if true and predicted groups essentially match. However, unlike many other cluster indices (e.g., the Rand index), it does not penalize if some of the groups are split up further. This property is particularly useful when assessing recursive partitions that might need several splits to form a certain group. Note that for the LRTs, we do not simulate the Cramér coefficient but simply determine its theoretical value assuming a given cutoff of zero in either one of $x_{1}, x_{2}$, or $x_{3}$ alone.

\section{Results}

Figure 4 displays the simulated power of the MPT tree in comparison to LRTs based on $x_{1}$ or $x_{2}$ as a function of the magnitude of parameter instability $(\delta)$ and sample size (n). In the first row, the results for the scenario are shown where the true cutoffs coincide with the medians of $x_{1}$ and $x_{2}$, respectively. Thus, the LRT that splits at the median of $x_{1}$ performs best for all magnitudes and sample sizes as it tests for the correct split of the root node. The MPT tree performs second best (except for very small magnitudes $\delta$ ), although it neither knows which variable $\left(x_{1}, x_{2}\right.$ or $\left.x_{3}\right)$ nor which cutoff point is correct. Furthermore, under the null hypothesis of homogeneous parameters $(\delta=0)$, the MPT tree holds its nominal significance level of $5 \%$; it does not

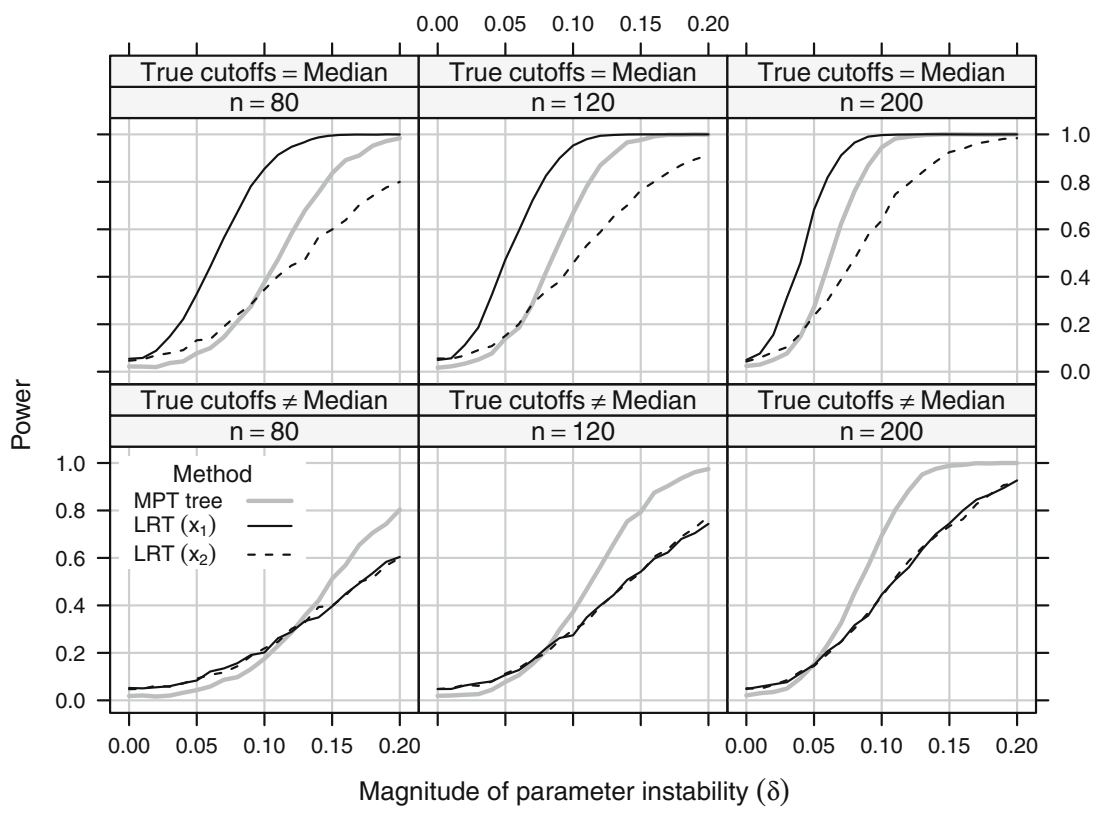

Fig. 4 Simulated power as a function of the magnitude of parameter instability $(\delta)$, sample size $(n)$, and the method used to test for instability. For the likelihood ratio tests (LRT), the median of $x_{1}$ or $x_{2}$, respectively, is used that either coincides with the true cutoff (upper panels) or not (lower panels) 
exceed $\alpha$ although it tests for instability in three variables. It is, however, somewhat conservative, especially for small sample sizes $n$, due to the asymptotic nature of the tests employed. Finally, the LRT that splits at the median of $x_{2}$ performs worst among the three methods despite using the correct split in one of the relevant variables. In the second row, where the true cutoffs do not coincide with the medians, the power of all methods goes down because the groups are more unbalanced (see above) and, more importantly, the search for the correct variables and cutoffs in the MPT tree pays off. This advantage of the MPT tree over the LRTs becomes more pronounced for larger magnitudes of parameter instability and larger sample size.

In summary, because the MPT tree always determines the cutoffs in a data-driven way, it cannot profit from "knowing" the true cutoffs in contrast to the LRTs. Therefore, the latter tests will have a power advantage over MPT trees if the true cutoff and the relevant variables are used. Conversely, when the cutoffs are unknown, the MPT tree has an advantage over LRTs, which depend on an often arbitrary choice of the cutoff (here, the median).

The second part of the results shows the accuracy of the MPT tree in recovering the true partitions. Figure 5 displays the average Cramér coefficient of agreement between true and predicted group membership as a function of the magnitude of parameter instability $(\delta)$ and sample size $(n)$. In both cutoff scenarios, the Cramér coefficient of the MPT tree increases with increasing parameter instability and sample size; however, it is generally somewhat lower in the second scenario in the right panel. This is due to the fact that the groups 1 and 3, which differ from the middle group 2,

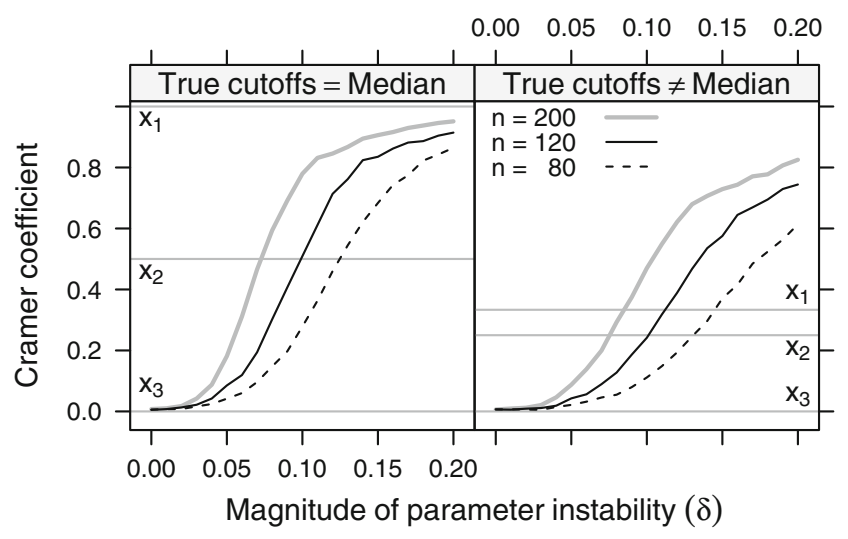

Fig. 5 Average Cramér coefficient of agreement between true and MPT-tree-predicted group membership as a function of the magnitude of parameter instability $(\delta)$ and sample size $(n)$. Horizontal lines indicate the Cramér coefficient when splitting the sample along the median of $x_{1}, x_{2}$, or $x_{3}$, which may either be the true cutoff (left panel) or not (right panel). As $x_{3}$ is unrelated to the groups, its Cramér coefficient is zero are smaller and hence harder to detect. As a reference, both panels show the theoretical Cramér coefficient of the deterministic splits using the medians of $x_{1}, x_{2}$, and $x_{3}$, respectively. For the split in $x_{3}$, this is generally 0 because this split is completely unrelated to the true groups in either scenario. For a split at the median of $x_{1}$ in the first scenario, the Cramér coefficient is 1 because this exactly catches the first split of the tree (and ignoring the second split is not penalized by the Cramér coefficient). However, if the true cutoff in $x_{1}$ differs from the median, the theoretical Cramér coefficient drops to $1 / 3$. Similarly, the Cramér coefficient for the deterministic split at the median of $x_{2}$ yields $1 / 2$ if this coincides with the true cutoff, and $1 / 4$ otherwise. Thus, in both scenarios, the Cramér coefficient of the MPT tree approaches the best possible value of 1 only for large $\delta$ and/or $n$; however, in the second scenario, this outperforms the deterministic splits already for values of $\delta$ above around 0.1 (depending on the sample size).

In conclusion, these results show that subgroups previously defined on the covariates are satisfactorily recovered by recursive partitioning based on an MPT model. In contrast to the likelihood ratio test, neither the relevant covariates nor the cutpoints have to be known in advance. A limitation of the results presented here is that they were obtained for a single MPT model (the 1HT model) and two similar tree structures (cutoff scenarios). Nevertheless, similar results can be obtained in other setups (see references cited above). Hence, we believe that these insights contribute evidence that MPT trees constitute a useful tool for detecting parameter heterogeneity in realistic settings.

\section{Simulation study 2: MPT trees versus latent-class models}

To assess how different heterogeneity detection methods based on the same MPT model compare, we investigate MPT trees in contrast with latent-class MPT (mixture) models (Klauer, 2006). As in the previous section, the focus is restricted to a single MPT model under a number of realistic settings. The procedure follows earlier studies, which include the comparison of model-based trees and mixture models for linear regression (Frick et al., 2014a) and for the Bradley-Terry-Luce choice model (Frick, Strobl, \& Zeileis, 2014b). While both the trees and the latent-class models are based on the same MPT model and aim at detecting subgroups with homogeneous parameters, they differ in several respects:

- For a mixture model, the number of groups has to be fixed before estimating parameters. Subsequently, the number of groups is often chosen based on information criteria or sequential tests. In contrast, for a tree the 


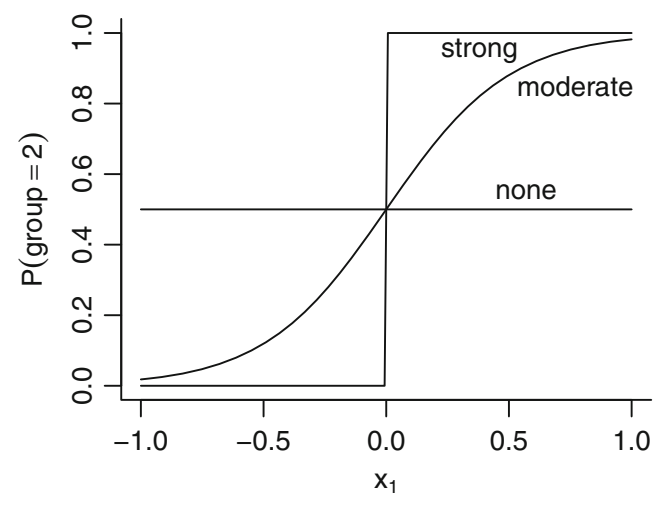

Fig. 6 Probability of membership in the second group as a function of covariate $x_{1}$ and strength of association between $x_{1}$ and the groups. The depicted settings are used in the second simulation study

number of groups is determined recursively from application of parameter instability tests, where in each step only a single MPT model has to be estimated.

- Mixture models ignore any covariates, but detect latent subgroups based on the response variable only. Trees require informative covariates: If no covariates associated with the subgroups are available, the groups cannot be detected. The selection of relevant covariates, however, is inherent to trees.

- Trees yield a hard clustering of the observations, mixtures a probabilistic clustering. The sample splits of a tree represent abrupt shifts in parameter values. Multiple splits in a covariate are able to represent a non-monotonic transition.

The following simulation investigates how these differences between the two methods affect their ability to detect parameter heterogeneity.

\section{Simulation design and experimental settings}

The design and settings are identical to those in the previous section apart from the following changes: A two-group 1HT model is employed for data generation, where $\Theta=$ ( $r_{\text {group }}, b=.2$ ) for group $\in\{1,2\}$. Only covariate $x_{1}$ is related to the groups as explained below; $x_{2}$ and $x_{3}$ are noise variables. The magnitude of parameter instability is controlled by the deviation $\delta \in\{0, .01, .02, \ldots, .16\}$ from the average recognition probability $r=.5$, where the groupspecific recognition probabilities are $r_{1}=.5-\delta$ and $r_{2}=.5+\delta$. The sample size $(n=120)$ is constant in all simulations.

The strength of the association between the covariate $x_{1}$ and the two groups is manipulated via the logistic regression model

$\log \frac{P(\text { group }=2)}{P(\text { group }=1)}=\beta \cdot x_{1}$, which predicts the probability of membership in the second group. The regression coefficient $\beta$ is set to 0,4 , and $\infty$, which we label no, moderate, and strong association with $x_{1}$, respectively (see Fig. 6). The first setting $(\beta=0)$ renders $x_{1}$ a noise variable; the second $(\beta=4)$ represents a smooth transition of group membership as a function of $x_{1}$; the third setting $(\beta=\infty)$ represents a step function so that group membership abruptly shifts from 1 to 2 when $x_{1}$ becomes positive.

In summary, for each of the three strengths of association and each magnitude of parameter instability, 500 data sets are generated to compute the outcome measures below for MPT trees and MPT mixture models. We expect that higher magnitudes $\delta$ will improve the detection performance of both trees and mixture models. For MPT trees, however, this should interact with the strength of association between the covariate $x_{1}$ and the group membership: The stronger this association, the better is the performance of MPT trees. For MPT mixture models, the strength of association should not affect their performance since they detect groups based on only the response frequencies.

\section{Outcome measures}

As in the previous section, we consider as outcome measures (1) the power to reject the null hypothesis of parameter stability and (2) the Cramér coefficient for classification accuracy. For the mixture models, however, the decision of how many latent groups are detected is based on the Bayesian information criterion (BIC) rather than on a significance test. Accordingly, we define the hit rate (instead of power) as the proportion of cases in which a mixture model with two or more groups is selected based on BIC.

\section{Results}

Figure 7 shows the results for both power and hit rate (upper panels) and Cramér coefficient (lower panels) for both methods. For the MPT tree, the influence of the magnitude of parameter instability $(\delta)$ on its power strongly depends on the strength of association between the covariate $x_{1}$ and the groups: If $x_{1}$ is unrelated (left panel), power is essentially zero. If, however, $x_{1}$ is moderately or strongly associated with the groups, power quickly rises with magnitude $\delta$, and the tree outperforms the mixture model already for small values of $\delta$. For the mixture model, on the other hand, the strength of association between $x_{1}$ and the groups is irrelevant for $\delta$ 's influence because it ignores information in the covariates: As soon as $\delta$ is sufficiently large, the mixture model detects latent groups; therefore, the pattern is the same in all three upper panels of Fig. 7.

For the classification accuracy as measured by the Cramér coefficient (lower panels), the results are similar to 


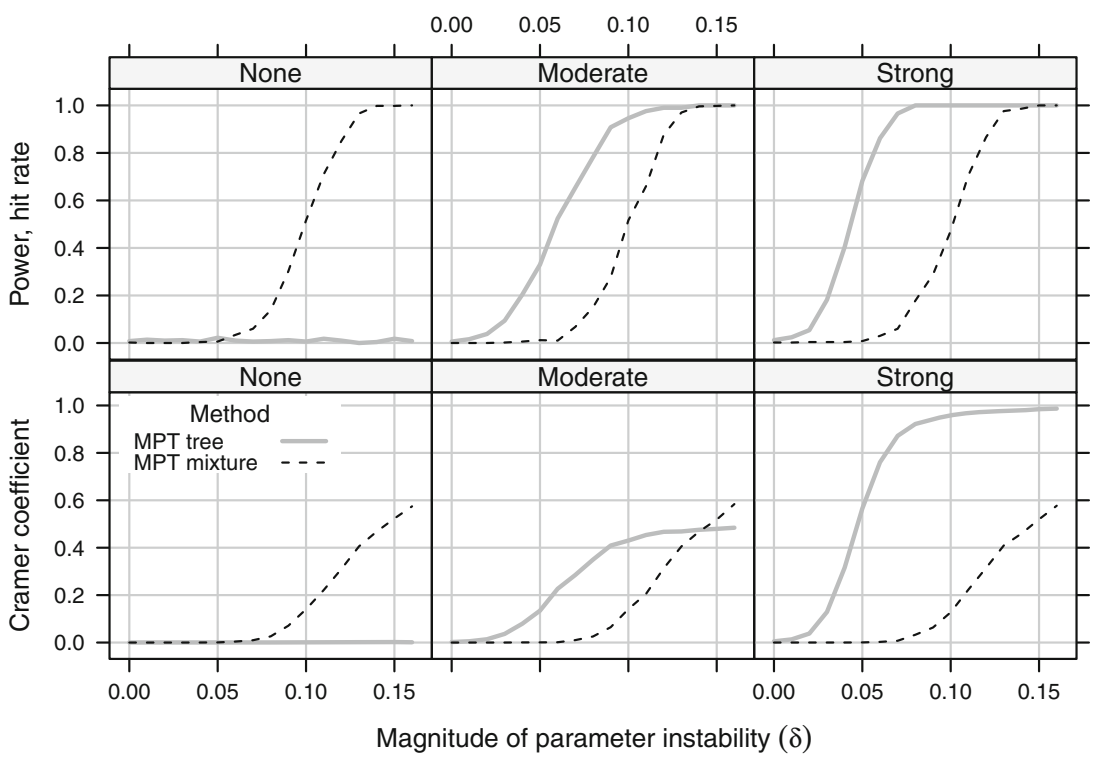

Fig. 7 Simulated power and hit rate (upper panels) and average Cramér coefficient (lower panels) as a function of the magnitude of parameter instability $(\delta)$, the strength of association between the covariate and the groups, and the method used to detect the instability

power and hit rate. For the MPT tree, the results depend on the strength of association between $x_{1}$ and the groups; for the mixture model, this association is irrelevant. If there is no association (left panel), the tree cannot correctly classify the observations since it does not detect any groups in the first place. As the association becomes stronger, the MPT tree's classification accuracy becomes better. For moderate association (middle panel), the tree has higher classification accuracy than the mixture model for $\delta<0.13$; for larger magnitudes, the mixture model becomes better. For the strongest association (right panel), the MPT tree outperforms the mixture model over the entire range of $\delta$.

Comparing the middle and right panels of Fig. 7 illustrates the detection and classification mechanism inherent in MPT trees: For smooth transitions between the groups, the tree can only approximate the groups by splits in the covariates. For abrupt transitions, the tree is most successful in finding the true groups. The problem of approximating smooth transitions through several split points, however, is relevant only for numeric covariates. For categorical covariates, the tree can only split observations into groups using the corresponding categories and does not have to select split points anyway.

In conclusion, these results show that the main factor determining the relative performance of MPT trees and mixture models is the availability of covariates that explain the heterogeneities. If such covariates are available, MPT trees are able to detect less pronounced group differences. In contrast, MPT mixture models cannot directly leverage such covariate information if it is available. However, if no covariates can explain the parameter heterogeneities, MPT mixture models can still identify sufficiently distinct groups of observations while MPT trees lack power. In practice, it is likely that situations in between the two extremes occur and

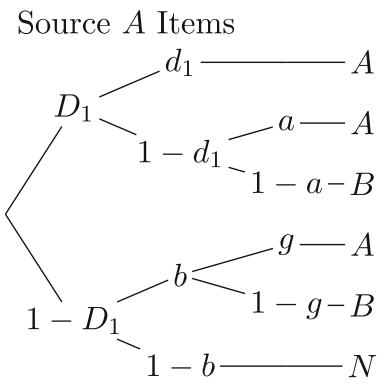

Fig. 8 Graph of the MPT model for the source monitoring paradigm (Batchelder \& Riefer, 1990). Latent cognitive processes are: detectability of Source $A$ items $\left(D_{1}\right)$, detectability of Source $B$ items $\left(D_{2}\right)$, source discriminabilities for Source $A\left(d_{1}\right)$ and Source $B$ items
Source $B$ Items $\quad$ New Items

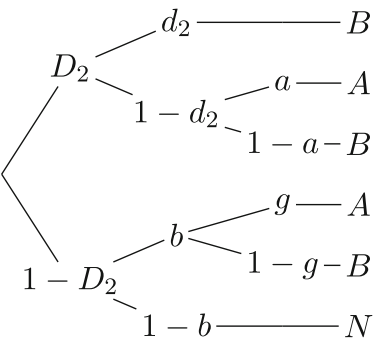

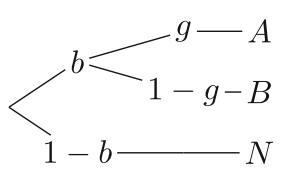

$\left(d_{2}\right)$, bias for responding "old" to a nondetected item $(b)$, guessing that a detected but nondiscriminated item belongs to Source $A(a)$, and guessing that the item is a Source $A$ item $(g)$ 
that results depend on the differences between the groups (in the simulation measured by $\delta$ ) and the strength of association between covariates and groups (in the simulation measured by $\beta$ ). Thus, if fitting an MPT tree does not lead to any subgroups, it would still be advisable to check for subgroups using MPT mixture models.

\section{Two applications}

This section covers two applications of recursive partitioning based on MPT models. The first analyzes a new data set for which the potential partitions were unknown a priori (as in most applications) but were the primary research interest. The second is a reanalysis of a published data set (Riefer, Knapp, Batchelder, Bamber, \& Manifold, 2002), where the focus is on how well the MPT tree succeeds in uncovering the a priori hypothesized partitions.

\section{Source monitoring}

The first application considers a typical source monitoring experiment: Participants study two lists of items as presented by either Source $A$ or Source $B$. Afterwards, in a memory test, participants are shown old and new items intermixed and asked to classify them as either $A, B$, or new $(N)$.

Figure 8 displays the MPT model for the source monitoring paradigm by Batchelder and Riefer (1990). To illustrate, consider the paths from the root to an $A$ response for a Source $A$ item (left tree in the figure). With probability $D_{1}$, a respondent detects an item as old. If, in a second step, he or she is able to discriminate the item from a Source $B$ item $\left(d_{1}\right)$, then the response will correctly be $A$; else, if discrimination fails $\left(1-d_{1}\right)$, a correct $A$ response can only be guessed with probability $a$. If the item was not detected as old in the first place $\left(1-D_{1}\right)$, the response will be $A$ only if there are both a response bias for "old" $(b)$ and a guess for the item being Source $A(g)$. The remaining paths in the left tree lead to classification errors $(B, N)$. The middle and right trees in Fig. 8 represent processing of Source $B$ and new items, respectively.

Such a source-monitoring experiment was conducted at the Department of Psychology, University of Tübingen. The sample consisted of 128 participants (64 female) aged between 16 and 67 years. Two source conditions were used in the study phase: Half of the respondents had to read the presented items either quietly (think) or aloud (say). The other half wrote them down (write) or read them aloud (say). Items were presented on a computer screen at a self-paced rate. In the final memory test, studied items were mixed with new distractor items, and respondents had to classify them as either $A, B$, or new by pressing a button on the screen.
The response frequencies are analyzed using the above MPT model for source monitoring (Figure 8; Batchelder $\&$ Riefer, 1990), where $a=g$ is assumed for identifiability. In addition, discriminability is assumed to be equal for both sources $\left(d_{1}=d_{2}\right)$ as in a similar example in Batchelder and Riefer (1990). As a research question, we investigate whether there are any effects of source condition, gender, or age on the model parameters. The MPT tree uses a Bonferroni-corrected significance level of $\alpha=0.05$ and a minimum number of five participants per node.

Figure 9 shows the tree resulting from recursive partitioning of the source monitoring MPT model. The node numbers are labels assigned from left to right, starting from the top, used to identify a given node. Table 1 displays the results of the parameter instability tests for every node. In Node 1, the full sample, only source type is significant, $S=28.48, p<.001$, so it is selected for splitting; since it is a binary variable, no cutpoint has to be computed. For the think-say subgroup in Node 2, age is selected for splitting, $S=20.77, p=.043$, and the optimal cutpoint is found at age 46 . No further parameter instability is detected in the subgroups, so the procedure stops. The fact that gender is never selected as the splitting variable suggests that there is no significant parameter heterogeneity with respect to gender.

Table 2 contains the resulting three sets of parameter estimates and the response proportions for each end node of the tree. The estimates reflect the combined influence of the covariates. For the think-say sources (Nodes 3 and 4 in Fig. 9 and in Table 2), $D_{2}$ exceeds $D_{1}$ indicating an advantage of say items over think items with respect to detectability. For the write-say sources (Node 5), $D_{2}$ and $D_{1}$ are about the same indicating that for these sources no such advantage exists. The think-say subgroup is further split by

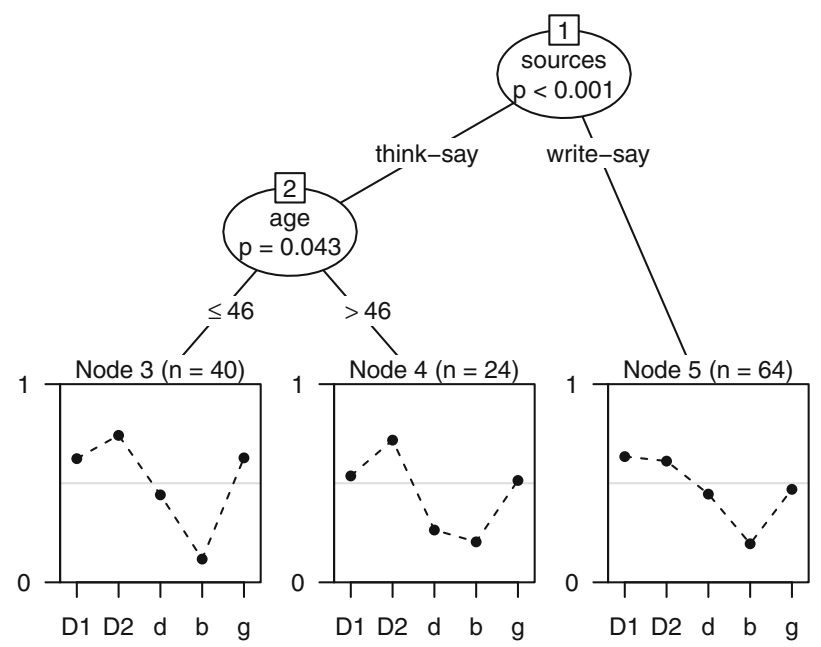

Fig. 9 Partitioned MPT model for source monitoring data indicating that parameters vary with combinations of the covariates source type and age 
Table 1 Parameter instability test statistic $(S)$ and Bonferroniadjusted $p$ value for each covariate per node (see Fig. 9)

\begin{tabular}{|c|c|c|c|c|c|c|}
\hline \multirow[b]{2}{*}{ Node } & \multicolumn{2}{|c|}{ Sources } & \multicolumn{2}{|l|}{ Age } & \multicolumn{2}{|c|}{ Gender } \\
\hline & $S$ & $p$ & $S$ & $p$ & $S$ & $p$ \\
\hline 1 & 28.48 & .000 & 16.93 & .249 & 9.00 & .292 \\
\hline 2 & - & - & 20.77 & .043 & 2.84 & .924 \\
\hline 3 & - & - & 10.25 & .763 & 4.28 & .760 \\
\hline 4 & - & - & 8.59 & .822 & 5.46 & .593 \\
\hline 5 & - & - & 8.06 & .965 & 7.41 & .347 \\
\hline
\end{tabular}

Note: Significant test results are in bold face.

age with the older participants having lower values on $D_{1}$ and $d$, which suggests lower detectability of think items and lower discriminability as compared to the younger participants. This age effect seems to depend on the type of sources as there is no such effect for the write-say sources. In addition, there are only small effects for the bias parameters $b$ and $g$, which are psychologically less interesting in this application. The effects of the covariates can also be seen for the response proportions (Table 2), albeit less clearly than for the parameter estimates: The proportions of a correct response $\left(p_{1}, p_{5}, p_{9}\right)$ are slightly lower in Node 4 than in the other nodes, corresponding to the lower $D_{1}$ and $d$ values.

The significance tests within MPT trees are global tests for parameter heterogeneity. A significant test indicates that at least one parameter is not constant across groups. In order to facilitate interpretation, it may help to graphically inspect the parameter estimates and their $95 \%$ confidence intervals. In the left panel of Fig. 10, the $d$ parameter is lower in Node 4 than in the other groups; similarly the $b$ parameter is lower and the $g$ parameter is higher in Node 3 than in the other groups. In addition to this graphical assessment of parameter-wise differences, one could also try to conduct formal post hoc tests. However, we do not do so for two reasons: (1) We already have conducted sequential global tests in the construction of the tree, and subsequent post hoc comparisons would not have to be in sync with these. (2) Adjusting post hoc tests after model selection in finite samples is challenging and to our knowledge no widely accepted procedure exists for tree-based model selection (for a discussion see Merkle \& Zeileis, 2013; Zeileis \& Hornik, 2007). If formal confirmatory inference in the detected subgroups is of prime interest, it would be advisable to gather additional experimental data based on the insights from the MPT trees.

\section{Storage-retrieval model for pair-clustering data}

Riefer et al. (2002) report a study on memory deficits in schizophrenic $(n=29)$ and organic alcoholic $(n=21)$ patients, who were compared to two matched control groups $(n=25, n=21)$. Participants were presented with 20 pairs of semantically related words. In a subsequent memory test, they freely recalled the presented words. This procedure was repeated for a total of six study and test trials. Responses were classified into four categories: each pair is recalled adjacently $\left(E_{1}\right)$, each pair is recalled non-adjacently $\left(E_{2}\right)$, one word in a pair is recalled $\left(E_{3}\right)$, neither word in a pair is recalled $\left(E_{4}\right)$. Riefer et al. (2002) analyzed the data using the storage-retrieval model for pair clustering (Batchelder and Riefer, 1986) displayed in Fig. 11. This model aims at separately measuring storage and retrieval capacities of episodic memory by its parameters $c$ and $r$. Here, we reanalyze the response frequencies using an MPT tree; the tree employs a Bonferroni-corrected significance level of $\alpha=$ 0.10 in order to visualize marginally significant splits.

Figure 12 shows the results of the recursive partitioning based on the storage-retrieval model. Table 3 contains the parameter estimates associated with the end nodes of the MPT tree and the response proportions in each of the four categories $\left(E_{1}\right.$ to $\left.E_{4}\right)$. The first split of the tree separates the two patient and control groups. In the control groups, the parameters improve with repeated presentation of the items: In Node 5, trial is selected as splitting variable, and the optimal cutpoint is $\leq 2,>2$. Within the $\leq 2$ partition, there is again a split into $\leq 1,>1$. All three parameters constantly increase for one, two, and more than two presentations; the increase is particularly pronounced for the $r$ parameter. The patient groups, on the other hand, do not improve to the same extent. Indeed, their improvement over trials is so weak that it does not attain significance. Neither storage $(c)$ nor retrieval $(r)$ parameters for these groups on average reach the level of the control groups. Marginally

Table 2 Maximum likelihood estimates of source monitoring model parameters and response proportions associated with the end nodes of the MPT tree in Fig. 9

\begin{tabular}{|c|c|c|c|c|c|c|c|c|c|c|c|c|c|c|}
\hline Node & $D_{1}$ & $D_{2}$ & $d$ & $b$ & $g$ & $p_{1}$ & $p_{2}$ & $p_{3}$ & $p_{4}$ & $p_{5}$ & $p_{6}$ & $p_{7}$ & $p_{8}$ & $p_{9}$ \\
\hline 3 & .62 & .74 & .44 & .12 & .63 & .13 & .04 & .08 & .07 & .13 & .06 & .04 & .02 & .44 \\
\hline 4 & .54 & .72 & .26 & .20 & .51 & .09 & .06 & .09 & .07 & .12 & .06 & .06 & .04 & .40 \\
\hline 5 & .63 & .61 & .45 & .19 & .47 & .12 & .05 & .07 & .05 & .12 & .08 & .04 & .05 & .40 \\
\hline
\end{tabular}

Note: $p_{1}$ to $p_{9}$ refer to the response categories $A, B$, and $N$ for Source $A$, Source $B$, and new items, respectively (see Fig. 8) 

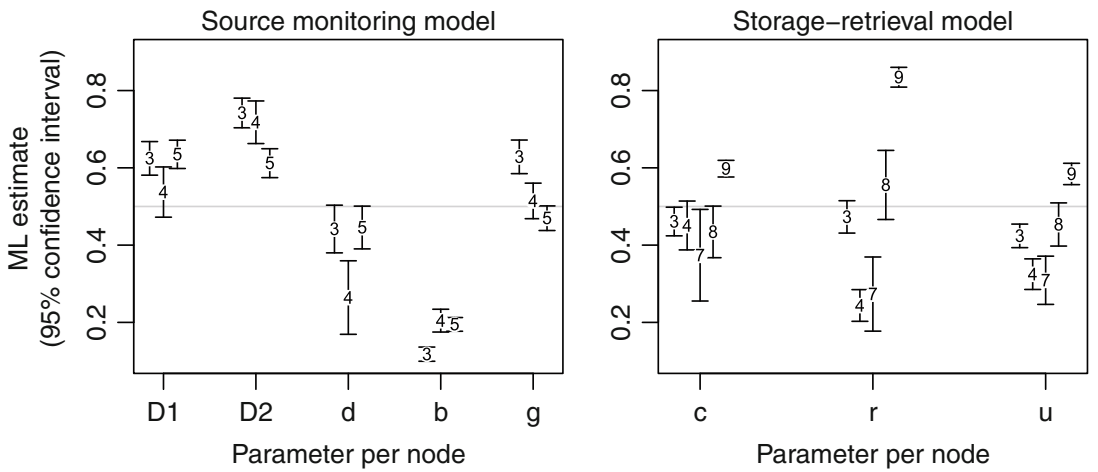

Fig. 10 Parameter estimates and 95\% confidence intervals for the source monitoring (left panel) and storage-retrieval models (right panel). The numbers refer to the end nodes of the MPT trees in Figs. 9 and 12, respectively

significant (Node 2) is the difference between schizophrenic and organic alcoholic patients: While these groups are comparably weak at storing new information, the retrieval is even more impaired in the organic alcoholic patients.

The results of our MPT tree analysis of the data are consistent with the findings in Riefer et al. (2002). The main conclusions is that alcoholic patients with organic brain damage exhibit essentially no improvement in retrieval over trials. Schizophrenic patients improve, albeit less than the control patients, in both storage and retrieval capacities. These results are also reflected by the response proportions. The proportion of recalling both items in a pair adjacently $\left(p_{1}\right)$ or non-adjacently $\left(p_{2}\right)$ increases for the control groups (Nodes 7, 8, and 9) while the proportion of failing to recall any item $\left(p_{4}\right)$ decreases. For schizophrenic patients (Node 3), the improvement is only modest and on average only reaches the level of the control patients after one trial of practice. For the alcoholic patients with organic brain damage (Node 4), there is no improvement with $p_{1}$ and $p_{2}$ staying at the lowest and $p_{4}$ staying at the highest value throughout.

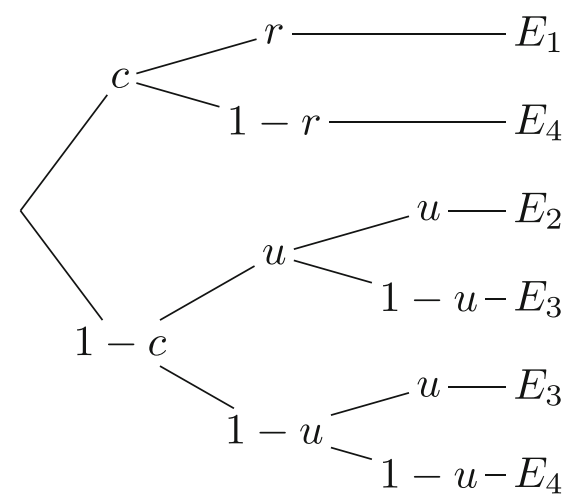

Fig. 11 Graph of the storage-retrieval model for pair clustering (Batchelder \& Riefer, 1986). Latent cognitive processes are: forming a cluster for a category pair $(c)$, successful retrieval of a stored cluster $(r)$, and storage/retrieval of an unclustered item $(u)$
The right panel of Fig. 10 shows the parameter estimates and $95 \%$ confidence intervals to facilitate their comparison. Clearly visible are the ascending pattern of all three parameters in the control groups (Nodes 7, 8, and 9) as well as the low $r$ parameter estimate for the alcoholic patients with organic brain damage.

Other than in the first application, partitioning is done here between observations, not between participants. Each participant contributes six response vectors, one for each trial, to the data set. Consequently, responses from the same participant may appear in more than a single end node. In order to account for the clustering of the responses contributed by the same person, a clustered covariance matrix estimate $\hat{I}$ for the maximum likelihood scores in Eq. 13 is employed in the instability tests. Generally, in situations with clustered data, the parameter instability tests within the tree should be considered with care. In the present application, the resulting tree structure is well in line with the hypothesized effects and the results of previous analyses (Riefer et al., 2002).

\section{Discussion}

We introduce MPT trees as a tool for investigating the effects of covariates on MPT model parameters. The core of MPT trees is model-based recursive partitioning (MOB), which recursively searches for covariates that induce parameter heterogeneity. When such a variable is found, a (locally) optimal cutpoint is detected and the sample is split. As a result, groups of participants are established with (approximately) the same model parameters. As has been illustrated by simulation and in the application examples, the groups do not have to be known beforehand, combinations of relevant covariates are identified, and interactions between covariates are incorporated automatically provided that suitable covariates exist. The general idea of MOB is not restricted to MPT models but has proved useful in other areas of 


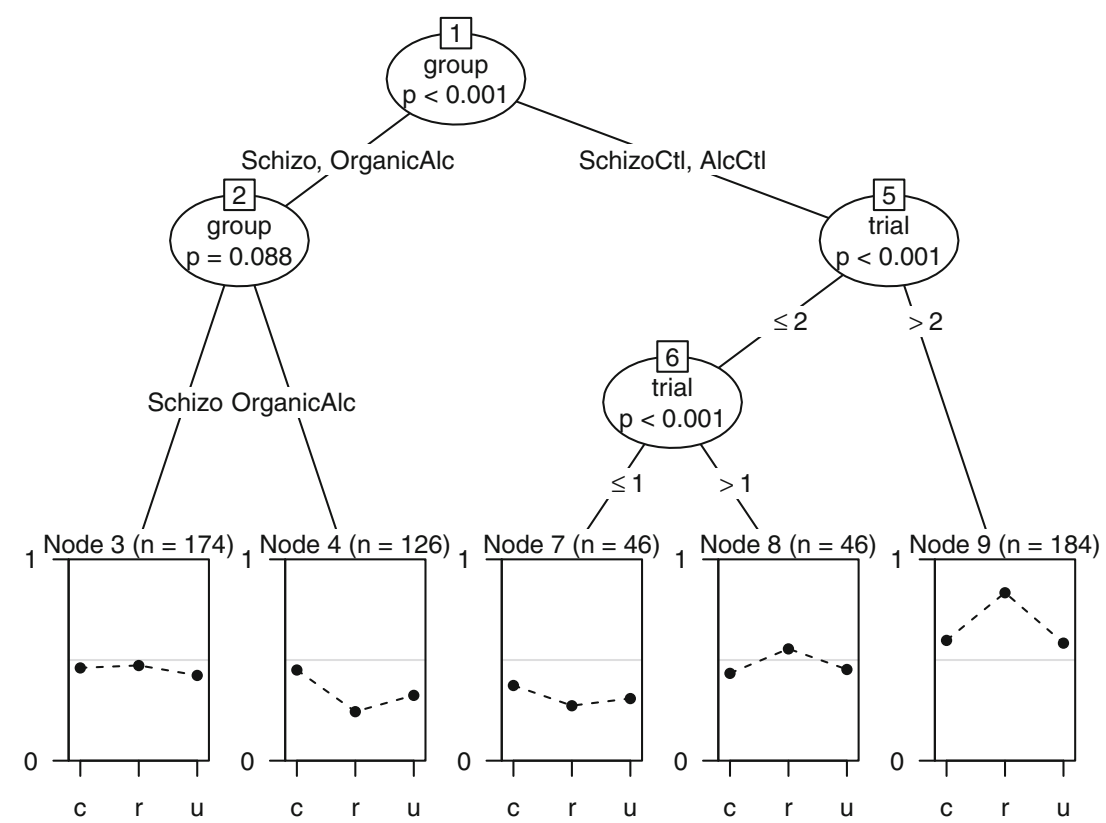

Fig. 12 Partitioned storage-retrieval model for pair-clustering data indicating that parameters vary with combinations of the covariates patient group and trial number

psychological modeling (Merkle and Zeileis, 2013; Strobl et al., 2015; Strobl et al., 2011). Therefore, it seems promising to further extend it to models where individual differences in parameters due to covariate effects need to be accounted for.

As shown in the second simulation study, successful application of MPT trees requires that covariates explaining the parameter heterogeneity exist. Also, there are patterns of dependence on the covariates against which the underlying parameter instability tests have more or less power. For example, Fig. 7 shows that power decreases with decreasing slope of a covariate. Another example that is often discussed in the tree literature is that power decreases for interaction effects in two covariates with almost no main effects. In this so-called XOR or chessboard problem, the simple

Table 3 Maximum likelihood estimates of storage-retrieval model parameters and response proportions associated with the end nodes of the MPT tree in Fig. 12

\begin{tabular}{llllllll}
\hline Node & $c$ & $r$ & $u$ & $p_{1}$ & $p_{2}$ & $p_{3}$ & $p_{4}$ \\
\hline 3 & .46 & .47 & .42 & .22 & .10 & .26 & .42 \\
4 & .45 & .24 & .32 & .11 & .06 & .24 & .59 \\
7 & .37 & .27 & .31 & .10 & .06 & .27 & .57 \\
8 & .43 & .56 & .45 & .24 & .12 & .28 & .36 \\
9 & .60 & .83 & .58 & .50 & .14 & .20 & .17 \\
\hline
\end{tabular}

Note: $p_{1}$ to $p_{4}$ refer to the response categories $E_{1}$ to $E_{4}$ (see Fig. 11) local forward search that most trees (and MOB) employ would need to be enhanced by explicitly including interactions in the search-either manually or more formally as in Kim and Loh (2001) —or by relaxing the Bonferroni correction for multiple testing to control the false discovery rate (Alvarez-Iglesias et al., 2017), or by carrying out a global optimization rather than a forward search (Grubinger et al., 2014). Finally, rather than searching for a single split in numerical covariates, one could also consider a split into three or more groups (Zeileis et al., 2008). However, capturing such multi-group splits by a sequence of binary splits also leads to consistent results (Chong, 1995).

There are a number of approaches that partly share the same goals with MPT trees, that is, accounting for individual differences in model parameters by covariate effects or explaining parameter heterogeneity in general. Most notably, such approaches include latent-class MPT models, latent-trait MPT models with random subject effects, and fully parameterized MPT models with covariates as fixed effects. In the remainder, similarities and differences of these methods to MPT trees will be discussed.

MPT trees share similarities with latent-class MPT (mixture) models (Klauer, 2006; Stahl \& Klauer, 2007). As with latent-class models, the sample is partitioned into a discrete number of groups within each of which parameter homogeneity holds, while between groups parameters differ. The difference between these two approaches to parameter heterogeneity is that latent-class models identify a previously specified number of groups on the basis of the response 
variables only. MPT trees, in contrast, identify an unknown number of groups based on splits in the available covariates. In doing so, the groups become immediately interpretable in terms of covariate effects and interactions. A caveat is that in MPT trees the parameter heterogeneity is entirely attributed to covariate effects. Thus, without predictive covariates, heterogeneity might go unnoticed. On the other hand, as has been shown in the second simulation study, trees have higher power than latent-class models to detect parameter differences if informative covariates are available. As latent-class MPT models, MPT trees assume homogeneity across items. This is sometimes considered a less problematic assumption than the assumption of subject homogeneity (Klauer, 2006); not least because the item material can be experimentally controlled, whereas differences between participants in cognitive processes are often the main focus of the study.

In contrast to models with a discrete number of classes, random-effects models represent heterogeneity in a continuous way. The beta MPT model (Smith \& Batchelder, 2010) uses independent beta hyperdistributions for the MPT parameters to account for individual differences. Similarly, the latent-trait MPT model (Klauer, 2010) uses probittransformed multivariate normal hyperdistributions to represent parameter heterogeneity induced by persons and accounts for correlation between parameters. Both models assume homogeneity of items but can be extended to deal with heterogeneity of persons and items. The crossed random effects extension of the latent-trait MPT model (Matzke et al., 2015) accounts for both sources of parameter heterogeneity simultaneously. For these random-effects models, parameter estimation and hypothesis testing is carried out in a Bayesian framework using Markov chain Monte Carlo sampling. Whereas random-effects models treat parameter heterogeneity by introducing nuisance variables and assumptions about their distributions, MPT trees seek to explain heterogeneity by covariate effects and interactions.

Alternatively to MPT trees, the effects of covariates can be directly incorporated as fixed effects into a parametric model using a specific link function that relates a linear predictor to model parameters. Examples of such an approach include models with logit link function in cultural consensus theory (Oravecz et al., 2015), logit-link MPT models (Coolin et al., 2015, 2016; Michalkiewicz et al., 2013), and probit-link hierarchical MPT models (Arnold, Bayen, \& Smith, 2015; Michalkiewicz, Arden, \& Erdfelder, 2016a; Michalkiewicz, Minich, \& Erdfelder, 2016b). Such models will have high power for detecting covariate effects if the model specification matches the true data-generating process. The main advantage of MPT trees over direct modeling becomes apparent when such a functional form of the covariate effects cannot be justified or is unknown a priori:
Because of its semi-parametric nature, an MPT tree is able to detect even nonlinear effects and interactions between covariates without the need of a fully parameterized model; while with direct modeling, such effects would have to be explicitly included. This flexibility with respect to the functional form and its straightforward graphical representation make MPT trees a useful tool for analyzing the effects of covariates in MPT models.

To summarize, recent methodological, statistical, and computational advances have produced a diversity of methods that account for parameter heterogeneity in MPT models. These methods can be broadly distinguished by whether (1) the heterogeneity-inducing variables are observed and (2) the form of the influence of these variables on the parameters is known. If the relevant variables are not observed, latent-class and latent-trait MPT models are promising candidates for capturing unobserved heterogeneity. If the variables are observed and the form of their influence is known, fully parameterized MPT models are applicable. If, however, the relevant variables are observed (plus potentially many irrelevant variables) but the form of their influence is unknown, MPT trees provide an elegant approach to detecting and explaining heterogeneity by means of subject covariates.

\section{Computational details and software}

MPT trees are implemented in the mpttree() function in the psychotree package (Zeileis, Strobl, Wickelmaier, Komboz, \& Kopf, 2016b) for the R system for statistical computing (R Core Team, 2017). This function combines estimation of MPT models with mptmodel ( ) from psychotools (Zeileis, Strobl, Wickelmaier, Komboz, \& Kopf, 2016a) and model-based trees with mob () from partykit (Hothorn \& Zeileis, 2015). The mptmodel () function has been adapted from the mpt package (Wickelmaier \& Zeileis, 2011) in order to provide a lean and fast implementation of MPT models. An alternative implementation is within the MPTinR package (Singmann \& Kellen, 2013), which focuses more on model estimation and selection.

The empirical examples can be easily replicated using example ("mpttree", package = "psychotree" ). For the source monitoring example, the following $\mathrm{R}$ code produces the MPT tree displayed in Fig. 9:

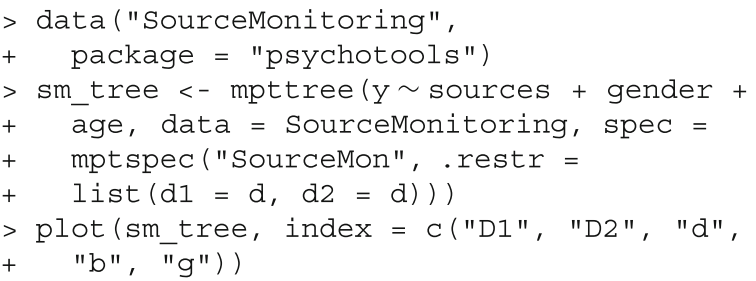


Parameter estimates in the end nodes of the tree and the parameter instability tests in the root node (Table 1) are obtained by:

$>$ coef (sm_tree)

$\begin{array}{rrrrrr} & \text { D1 } & d & g & b & \text { D2 } \\ 3 & 0.624 & 0.442 & 0.628 & 0.118 & 0.742 \\ 4 & 0.537 & 0.264 & 0.514 & 0.205 & 0.718 \\ 5 & 0.635 & 0.446 & 0.470 & 0.195 & 0.612\end{array}$

$>$ sctest.modelparty (sm_tree, node $=1$ )

$\begin{array}{lrrr} & \text { sources } & \text { gender } & \text { age } \\ \text { statistic } & 2.85 e+01 & 9.003 & 16.930 \\ \text { p.value } & 8.81 e-05 & 0.292 & 0.249\end{array}$

For the example on storage and retrieval deficits in psychiatric patients, this code creates the MPT tree in Fig. 11:

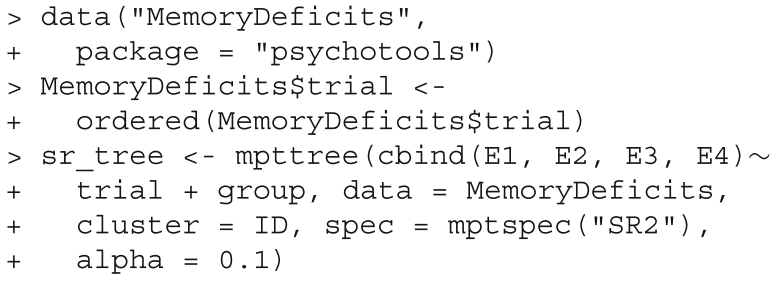

In this example, the trial variable is represented by an ordinal factor, and the Bonferroni-corrected significance level is $10 \%$. More information about the mpttree ( ) function is available in the package documentation via ?mpttree.

Our results were obtained using $\mathrm{R} 3.4 .0$ and $p s y$ chotree $0.15-1$, partykit 1.1-1, psychotools $0.4-2$, mpt $0.5-4$, and psychomix 1.1-4 (Frick, Strobl, Leisch, \& Zeileis, 2012); the latter package contains the mptmix ( ) function that implements latent-class MPT (mixture) models. $\mathrm{R}$ itself and all packages used are freely available under the terms of the General Public License from the Comprehensive R Archive Network (https://CRAN.R-project.org/).

Acknowledgments We would like to thank William H. Batchelder for making available the data on memory deficits in clinical subpopulations.

\section{References}

Agresti, A. (2002). Categorical data analysis. New York: Wiley. Akaike, H. (1974). A new look at the statistical model identification. IEEE Transactions on Automatic Control, 19, 716-723.

Alvarez-Iglesias, A., Hinde, J., Ferguson, J., \& Newell, J. (2017). An alternative pruning based approach to unbiased recursive partitioning. Computational Statistics \& Data Analysis, 106, 90-102.
Arnold, N. R., Bayen, U. J., \& Smith, R. E. (2015). Hierarchical multinomial modeling approaches: An application to prospective memory and working memory. Experimental Psychology, 62, 143152.

Batchelder, W. H., \& Riefer, D. M. (1986). The statistical analysis of a model for storage and retrieval processes in human memory. British Journal of Mathematical and Statistical Psychology, 39, 129-149.

Batchelder, W. H., \& Riefer, D. M. (1990). Multinomial processing models of source monitoring. Psychological Review, 97, 548-564.

Batchelder, W. H., \& Riefer, D. M. (1999). Theoretical and empirical review of multinomial process tree modeling. Psychonomic Bulletin \& Review, 6, 57-86.

Bishop, Y. M. M., Fienberg, S. E., \& Holland, P. W. (1975). Discrete multivariate analysis: Theory and practice. Cambridge: MIT Press.

Blackwell, H. R. (1963). Neural theories of simple visual discriminations. Journal of the Optical Society of America, 53, 129-160.

Chong, T. T.-L. (1995). Partial parameter consistency in a misspecified structural change model. Economics Letters, 49, 351-357.

Coolin, A., Erdfelder, E., Bernstein, D. M., Thornton, A. E., \& Thornton, W. L. (2015). Explaining individual differences in $\operatorname{cog}$ nitive processes underlying hindsight bias. Psychonomic Bulletin \& Review, 22, 328-348.

Coolin, A., Erdfelder, E., Bernstein, D. M., Thornton, A. E., \& Thornton, W. L. (2016). Inhibitory control underlies individual differences in older adults' hindsight bias. Psychology and Aging, 31, 224-238.

Erdfelder, E., Auer, T., Hilbig, B. E., Aßfalg, A., Moshagen, M., \& Nadarevic, L. (2009). Multinomial processing tree models: A review of the literature. Zeitschrift für Psychologie, 217, 108-124.

Fagan, J. F. (1984). Recognition memory and intelligence. Intelligence, 8, 31-36.

Frick, H., Strobl, C., Leisch, F., \& Zeileis, A. (2012). Flexible Rasch mixture models with package psychomix. Journal of Statistical Software, 48(7), 1-25.

Frick, H., Strobl, C., \& Zeileis, A. (2014a). To split or to mix? Tree vs. mixture models for detecting subgroups. In Gilli, M., González-Rodríguez, G., \& Nieto-Reyes, A. (Eds.) Processing of COMPSTAT 2014 - 21st international conference on computational statistics (pp. 379-386). Geneva: The International Statistical Institute/International Association for Statistical Computing.

Frick, H., Strobl, C., \& Zeileis, A. (2014b). To split or to mix? Uncovering group structures with trees and finite mixture models. Presented at the Psychoco 2014 International Workshop on Psychometric Computing, Tübingen, Germany. Abstract retrieved from http://www.psychoco.org/2014/abstracts/Frick_Strobl_Zeileis.txt

Grizzle, J. E., Starmer, C. F., \& Koch, G. G. (1969). Analysis of categorical data by linear models. Biometrics, 25, 489-504.

Grubinger, T., Zeileis, A., \& Pfeiffer, K.-P. (2014). evtree: Evolutionary learning of globally optimal classification and regression trees in R. Journal of Statistical Software, 61(1), 1-29.

Hothorn, T., \& Zeileis, A. (2015). partykit: A modular toolkit for recursive partytioning in R. Journal of Machine Learning Research, 16, 3905-3909.

Hu, X., \& Batchelder, W. H. (1994). The statistical analysis of general processing tree models with the EM algorithm. Psychometrika, 59, $21-47$.

Kim, H., \& Loh, W.-Y. (2001). Classification trees with unbiased multiway splits. Journal of the American Statistical Association, 96, 589-604.

Klauer, K. C. (2006). Hierarchical multinomial processing tree models: A latent-class approach. Psychometrika, 71, 7-31.

Klauer, K. C. (2010). Hierarchical multinomial processing tree models: A latent-trait approach. Psychometrika, 75, 70-98. 
Komboz, B., Strobl, C., \& Zeileis, A. (2016). Tree-based global model tests for polytomous Rasch models. Educational and Psychological Measurement.

Matzke, D., Dolan, C. V., Batchelder, W. H., \& Wagenmakers, E.-J. (2015). Bayesian estimation of multinomial processing tree models with heterogeneity in participants and items. Psychometrika, $80,205-235$.

Merkle, E. C., Fan, J., \& Zeileis, A. (2014). Testing for measurement invariance with respect to an ordinal variable. Psychometrika, 79, $569-584$.

Merkle, E. C., \& Zeileis, A. (2013). Tests of measurement invariance without subgroups: A generalization of classical methods. Psychometrika, 78, 59-82.

Michalkiewicz, M., Arden, K., \& Erdfelder, E. (2016a). Do smarter people make better decisions? The influence of intelligence on adaptive use of the recognition heuristic. Manuscript under review.

Michalkiewicz, M., Coolin, A., \& Erdfelder, E. (2013). Individual differences in use of the recognition heuristic. Presented at the meeting of the Society of Mathematical Psychology, Potsdam, Germany. Abstract retrieved from http://www.mathpsych. org/conferences/2013/

Michalkiewicz, M., Minich, B., \& Erdfelder, E. (2016b). Explaining individual differences in fast-and-frugal decision making. The impact of need for cognition and faith in intuition on use of the recognition heuristic. Manuscript under review.

Mirkin, B. (2001). Eleven ways to look at chi-squared coefficients for contingency tables. The American Statistician, 55, 111-120.

Oravecz, Z., Anders, R., \& Batchelder, W. H. (2015). Hierarchical Bayesian modeling for test theory without an answer key. Psychometrika, 80, 341-364.

Philipp, M., Zeileis, A., \& Strobl, C. (2016). A toolkit for stability assessment of tree-based learners. In Colubi, A., Blanco, A., \& Gatu, C. (Eds.) Proceedings of COMPSTAT 2016 - 22nd international conference on computational statistics (pp. 315325). Oviedo: The International Statistical Institute/International Association for Statistical Computing.

R Core Team. (2017). R: A Language and Environment for Statistical Computing. Vienna: R Foundation for Statistical Computing. https://www.R-project.org/

Riefer, D. M., \& Batchelder, W. H. (1988). Multinomial modeling and the measurement of cognitive processes. Psychological Review, 95, 318-339.

Riefer, D. M., \& Batchelder, W. H. (1991). Age differences in storage and retrieval: A multinomial modeling analysis. Bulletin of the Psychonomic Society, 29, 415-418.
Riefer, D. M., Knapp, B. R., Batchelder, W. H., Bamber, D., \& Manifold, V. (2002). Cognitive psychometrics: Assessing storage and retrieval deficits in special populations with multinomial processing tree models. Psychological Assessment, 14, 184 201

Schwarz, G. E. (1978). Estimating the dimension of a model. Annals of Statistics, 6, 461-464.

Singmann, H., \& Kellen, D. (2013). MPTinR: Analysis of multinomial processing tree models in R. Behavior Research Methods, 45, 560-575.

Smith, J. B., \& Batchelder, W. H. (2010). Beta-MPT: Multinomial processing tree models for addressing individual differences. Journal of Mathematical Psychology, 54, 167-183.

Stahl, C., \& Klauer, K. C. (2007). HMMTree: A computer program for latent-class hierarchical multinomial processing tree models. Behavior Research Methods, 39, 267-273.

Strobl, C., Kopf, J., \& Zeileis, A. (2015). Rasch trees: A new method for detecting differential item functioning in the Rasch model. Psychometrika, 80, 289-316.

Strobl, C., Wickelmaier, F., \& Zeileis, A. (2011). Accounting for individual differences in Bradley-Terry models by means of recursive partitioning. Journal of Educational and Behavioral Statistics, 36, $135-153$.

Swets, J. A. (1961). Is there a sensory threshold? Science, 134, 168177.

Wickelmaier, F., \& Zeileis, A. (2011). Multinomial processing tree models in R. Presented at the R User Conference, Coventry, UK. Retrieved from https://www.R-project.org/conferences/useR-2011/ TalkSlides/Contributed/17Aug_1705_FocusV_3-Psychometrics_1Wickelmaier.pdf

Zeileis, A., \& Hornik, K. (2007). Generalized M-fluctuation tests for parameter instability. Statistica Neerlandica, 61, 488508.

Zeileis, A., Hothorn, T., \& Hornik, K. (2008). Model-based recursive partitioning. Journal of Computational and Graphical Statistics, $17,492-514$

Zeileis, A., Strobl, C., Wickelmaier, F., Komboz, B., \& Kopf, J. (2016a). psychotools: Infrastructure for Psychometric Modeling. $\mathrm{R}$ package version $0.4-2$, https://CRAN.R-project.org/ package $=$ psychotools

Zeileis, A., Strobl, C., Wickelmaier, F., Komboz, B., \& Kopf, J. (2016b). psychotree: Recursive Partitioning Based on Psychometric Models. R package version 0.15-1, https://CRAN.R-project. org/package= psychotree 